\title{
Energy Efficient Hierarchical Cluster Head Election Using Exponential Decay Function Prediction
}

\author{
Ojoawo A.O and Adeniji O.D \\ Computer Science Department, University of Ibadan, Ibadan, Nigeria
}

\begin{abstract}
In the recent years, wireless sensor network (WSN) have witnessed increased interest in information gathering in applications such as combat field reconnaissance, security surveillance, environmental monitoring, patient health monitoring and so on. Thus, there is a need for scalable and energy-efficient routing, data gathering and aggregation protocols in these WSN environments. Various hierarchical clustering Protocols have been proposed by authors for WSN to improve system stability, lifetime, and energy efficiency. Clustering involves grouping nodes into disjoint and non-overlapping clusters. In this paper we motivate the need for clustering. Secondly, we present general classification of published clustering schemes. Thirdly, we review some existing clustering algorithms proposed for WSNs; highlighting their objectives, features, and so on. Finally, we develop an Average Energy (AvE) prediction algorithm using exponential decay function $y=A e^{-a x}+B$. We then combine this function with the probabilistic distributed LEACH of algorithm to determine suitable CHs. The combined algorithm was implemented on MATLAB simulator and tested for homogenous network. The result gathered from the simulation shows that the extended algorithm in homogenous network mode is able to achieve 39\% stability, $11 \%$ Average energy Dissipation per round and 40\% Lifespan better than LEACH-Homo. This paper proposes a new direction in improving energy efficiency of WSN routing protocol, which is desirable in some critical WSN applications. .
\end{abstract}

\section{KEYWORDS}

Wireless sensors networks, Clustering, Routing, sensor node, Average Energy, round, exponential decay curve

\section{INTRODUCTION}

Wireless Sensor Networks (WSNs) present relatively new generation of real time embedded systems with limited computational speed, energy and storage resources. WSNs are used where traditional networking infrastructure is impractical. [12] Different approaches and techniques have been proposed in the recent years for optimization of energy usage in Wireless Sensor Networks. WSN has also drawn the interest of researchers due its wide range of application such as combat field reconnaissance, security surveillance, environmental monitoring and so on. Sensors in these applications are expected to be deployed massively in a harsh or hostile environment, and are expected to operate autonomously. Advancement in miniaturized microelectronics devices made it possible to develop cheap low power micro-sensors for WSN.

DOI: $10.5121 /$ ijwmn.2018.10502 
Sensor node in a WSN has the ability to communicate with every other node wirelessly. A typical sensor node has these components: a radio transceiver with an antenna which has the ability to send or receive data packets, a microcontroller which could process data and schedule relative tasks, one or more sensors sensing the environment data, batteries providing energy supply, and a memory for data storage.

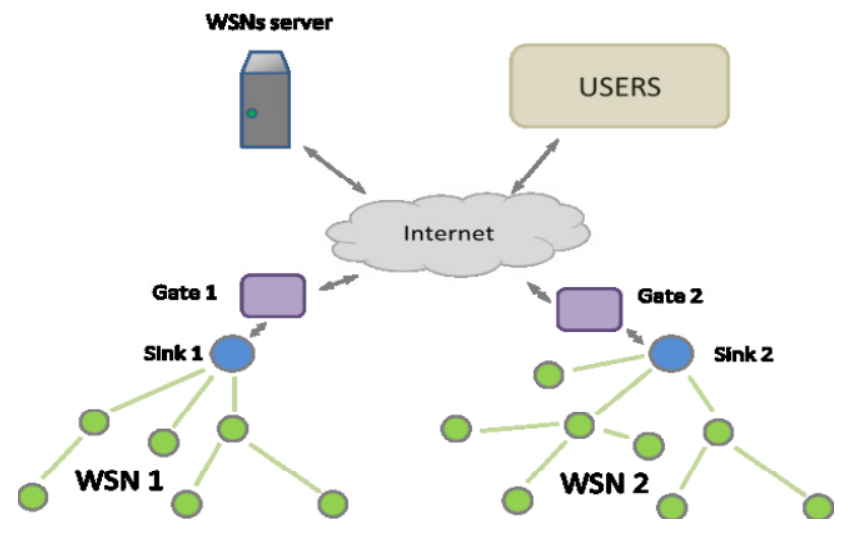

Figure 1. Wireless sensor network Architecture

\subsection{WHAT IS WirELESS SENSOR NETWORK?}

A Wireless Sensor Networks (WSNs) consist of spatially distributed self-configurable sensors. These sensors has the ability to monitor physical or environmental conditions, such as temperature, humidity, vibration, pressure, sound, motion and so on, with very low energy consumption. Sensed data collected by these nodes are sent to the base station or sink where further processing can take place. In most applications, only aggregated data are expected to be sent to the base station. In order to provide more accurate reports about their local regions, sensors in different regions of the field can collaborate to aggregate their data.

Since sensor nodes are deployed massively and are expected to operate unattended in condition where node maintenance is impractical. Therefore, energy efficiency and fault tolerance becomes the design goal in WSN.

A single sensor itself cannot gather useful information due to its limitation in term of its resources like power, CPU speed, and communication radio coverage and memory capacity. Massive deployment of sensor nodes is necessary to give room for collaboration in gathering information.

\subsection{Definition Of Some Common Terms Used In This Paper}

Sensor Node: A sensor node is the core component of a WSN. Sensor nodes can take on multiple roles in a network, such as simple sensing; data storage; routing; and data processing. 
Clusters: Clusters are the organizational unit for WSNs. The dense nature of these networks requires the need for them to be broken down into clusters to simplify tasks such a communication.

Clusterheads (CHs): Cluster heads are the organization leader of a cluster. They often are required to organize activities in the cluster. These tasks include but are not limited to dataaggregation and organization the communication schedule of a cluster.

Base Station: The base station is at the upper level of the hierarchical WSN. It provides the communication link between the sensor network and the end-user.

Routing: This is the transfer of data from source to destination through a path that will require minimum power cost.

\subsection{NEED For CluStering In WSN}

A typical WSN consists of hundreds to thousands of sensor nodes. Sensor nodes are energy constrained and are typically deployed in environments where recharging batteries is impractical. Therefore, it is obvious that specialized energy-aware routing and data gathering protocols offering high scalability should be applied in order increase the lifetime of the Network. Nodes in WSN are deployed in ad-hoc manner in large numbers and unaware of their location. Distributed clustering protocols that rely only on neighborhood information are preferred for WSNs. In most research work, it is assumed that there is a central location that is aware of the network topology. As it has been stated earlier that sensor nodes in WSN operate on batteries with limited energy, clustering approach is employed to achieve low message overhead to save energy. In addition, because nodes are deployed typically to and hash or hostile environment made them to be vulnerable to failure, thus there is a need for periodic re-clustering to heal disconnected regions and distribute energy consumption across all nodes. Periodic re-clustering is also needed because the parameters used in choosing $\mathrm{CH}$ are dynamic; values such as residual energy, centrality, node degree etc. changes overtime. The clustering techniques proposed for data processing typically consider static parameters, such as the distance between the nodes also assume that nodes are reliable which is not always true.

\subsection{CLUSTERING PROCESS}

To achieve energy efficiency and data aggregation, most researchers suggests sensors have to be partitioned into small groups called clusters. Each cluster has a coordinator, referred to as a cluster head $(\mathrm{CH})$, and a number of member nodes. Clustering forms a two level hierarchy where the $\mathrm{CH}$ is at the higher level and Member nodes at the lower level. The member nodes aggregate their sensed data to their respective $\mathrm{CH}$ and the $\mathrm{CH}$ forwards it to the base station. Because $\mathrm{CHs}$ transmit data often and over long distances, they use more energy compared to the member nodes, thus there is a need to rotate the $\mathrm{CH}$ role among the nodes on the network periodically for load distribution [6]. This process is usually referred to as re-clustering. In addition to Energy efficiency, clustering reduces channel contention and packet collisions, resulting in better network throughput under high load. 
International Journal of Wireless \& Mobile Networks (IJWMN) Vol. 10, No. 5, October 2018

\subsection{ADVANTAGES OF WSN CLUSTERING:}

1. Clustering reduces the size of the routing table stored at the individual nodes by localizing the route set up within the cluster.

2. Clustering can conserve communication bandwidth since it limits the scope of inter-cluster interactions to $\mathrm{CHs}$ and avoids redundant exchange of messages among sensor nodes.

3. The $\mathrm{CH}$ can prolong the battery life of the individual sensors and the network lifetime as well by implementing optimized management strategies.

4. Clustering cuts on topology maintenance overhead. Sensors would care only for connecting with their CHs.

5. A $\mathrm{CH}$ can perform data aggregation in its cluster and decrease the number of redundant packets.

6. A CH can reduce the rate of energy consumption by scheduling activities in the cluster.

\section{RELATED WORK}

Heinzelman, et.al [6] introduced a hierarchical clustering algorithm for sensor networks, called Low Energy Adaptive Cluster Hierarchy - based protocol (LEACH). In LEACH the operation is divided into rounds, during each round a different set of nodes are cluster-heads $(\mathrm{CH})$. Nodes that have been cluster heads cannot become cluster heads again for P rounds. Thereafter, each node has a $1 / \mathrm{p}$ probability of becoming a cluster head in each round. At the end of each round, each node that is not a cluster head selects the closest cluster head and joins that cluster to transmit data. $\mathrm{LEACH}$ probabilistic formula is given by:

$$
T(n)= \begin{cases}\frac{P}{1-P\left(\operatorname{rmod} \frac{1}{P}\right)}, & \text { if } n \in G \\ 0, & \text { otherwise }\end{cases}
$$

The cluster heads aggregate and compress the data and forward it to the base station, thus it extends the lifetime of major nodes. In this algorithm, the energy consumption will distribute almost uniformly among all nodes and the non-head nodes are turning off as much as possible. LEACH assumes that all nodes are in wireless transmission range of the base station which is not the case in many sensor deployments. In each round, LEACH has cluster heads comprising 5\% of total nodes. It uses Time Division Multiple Access (TDMA) as a scheduling mechanism which makes it prone to long delays when applied to large sensor networks. The figure below shows the communications in LEACH protocol. 


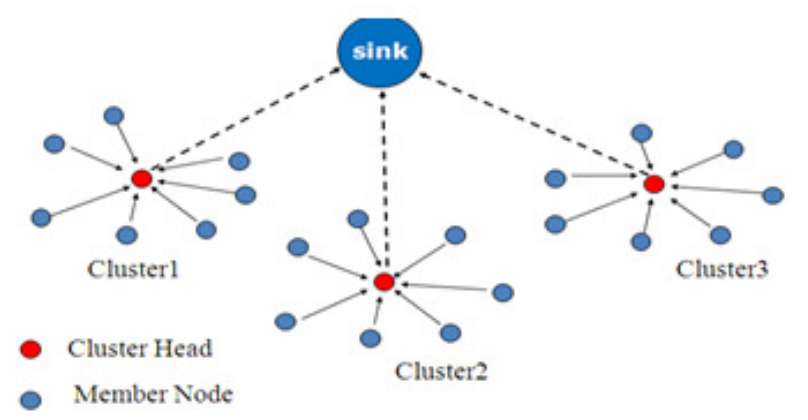

Figure 2. Hierarchical Routing Topology (Xuxun, 2012)

LEACH-C algorithm [3], each node sends their current location information and residual energy level to the sink. The sink in turn uses this information to select suitable $\mathrm{CH}$ based on their residual energy, centrality and so on. The major drawback of LEACH-C is that sensor nodes expend significant power sending this information of BS at each round. In addition, assumption that sensor nodes are equipped with GPS adds more complexity to the system.

LEACH protocol does not make use of high energy nodes to improve its stability when present, it only elongate the lifespan of the network, Stable Election Protocol (SEP) [7] however is a heterogeneous-aware protocol to prolong the time interval before the death of the first node (we refer to as stability period), which is crucial for many applications where the feedback from the sensor network must be reliable. SEP is based on weighted election probabilities of each node to become cluster head according to the remaining energy in each node. Simulation result shows that SEP always prolongs the stability period compared to (and that the average throughput is greater than) the one obtained using current clustering protocols. SEP protocol is sensitive to heterogeneity parameters capturing energy imbalance in the network. SEP yields longer stability region for higher values of extra energy brought by more powerful nodes. The demerit of SEP is that it is not suitable for homogeneous network.

In E-LEACH protocol [8] the cluster head selection is based on the residual energy level of the nodes. The residual energy level decides that whether the node will become a cluster head or not after the first round. In this protocol all nodes have equal probability to become the cluster head in the first round. The residual energy level in the second round is different for each node because of the first round communication. In this protocol the nodes that have a more energy level will become the cluster head rather than the nodes with low energy level. Therefore, this protocol improves the cluster head selection procedure. The drawback is that, it makes the network structure rigid, and may eventually lead to imbalance energy consumption on the network.

Another salient problem in LEACH is that the cluster head that is far away from base station require more energy to transfer the information to the base station and therefore it will die soon. To solve this problem, Two-Level LEACH (TL-LEACH) [1] protocol is proposed. In this protocol, the cluster head is responsible for collection and fusion of data like LEACH protocol from respective cluster members, but the cluster heads will not directly forward the data to the base station. It uses one of the cluster head that lies between cluster head and base station as a relay station. Disadvantage is the cluster heads closer to BS now has additional responsibility of 
helping other cluster heads to forward their data to BS, which eventually lead to hotspot problems.

DEEC (Distributed Energy Efficient Clustering) In DEEC [12] protocol all nodes use the initial and residual energy level to define the cluster heads. DEEC estimate the ideal value of network lifetime to compute the reference energy that each node should expend during each round. In a two-level heterogeneous network, where we have two categories of nodes, $\mathrm{m}$. $\mathrm{N}$ advanced nodes with initial energy equal to $E_{0} \cdot(1+a)$ and $(1-m) . N$ normal nodes, where the initial energy is equal to Eo. Where a and $\mathrm{m}$ are two variable which control the nodes percentage types (advanced or normal) and the total initial energy in the network $\mathrm{E}_{\text {total }}$.

LEACH-H (Hybrid Cluster Head Selection Leach) [9] was proposed in order to overcome the defect of short survival time and low degree of load balancing in case of LEACH. It uses the advantages of LEACH and LEACH-C. Cluster head is selected in the first round by base station in Leach-H, which effectively resolves the problem that the number of cluster head is uncertain in Leach. In the other rounds, the new cluster head used in the next round is selected in their own cluster by the current cluster head in Leach- $\mathrm{H}$, which resolves the issue of the dependence on the base station in Leach-C.

V-LEACH [2] protocol solves this problem by introducing the vice-cluster head. In V-LEACH protocol a cluster contains a cluster head, vice-cluster head and cluster nodes. In this protocol if the cluster head dies then vice-cluster head will start working as cluster head and the cluster head data will reach to the base station. There is no need to elect the new cluster head, so it will save the energy and enhance the network life time. LEACH-B [4] solves the problems, number of cluster heads and the ignorance of the node's residual energy, found in LEACH. This protocol adds a second selection of cluster heads to modify the number cluster-head in the set-up phase considering the node's residual energy per round. In order to save the energy consumption and to prolong the life span of the network, the protocol ensures that the partition of cluster is balance and uniform.

LEACH-DE [5] Improved LEACH Routing Protocol; It proposes selection of cluster head based on residual energy of a node and its position on the network. The average energy is estimated based on assumption that the sensor nodes have global knowledge of the network and are equipped with GPS. [14] EECP Energy efficient clustered protocol it uses the distance of the sensor from the sink as the major issue for the selection of a cluster in the sensor network. Improves Energy Efficiency of the network, drawback is that Significant overhead due to regular communication with the sink during estimation of the distance.

In order to save the energy consumption and to prolong the life span of the network, routing protocol must ensure that the partition of cluster is balance and uniform. U-LEACH [10] is an approach to address this problem. It describes a Uniform Distribution Technique (UDT) for selecting $\mathrm{CHs}$ and their corresponding clusters. 
International Journal of Wireless \& Mobile Networks (IJWMN) Vol. 10, No. 5, October 2018

\section{Development Of An Extended Cluster Head Selection AlgorithM}

In the LEACH hierarchical protocol, residual energy of the nodes selected as $\mathrm{CH}$ is not taking into consideration by the probabilistic algorithm $T(n)$ as $T(n)=p /\left(1-p\left(r^{*} \bmod (1 / p)\right)\right)$, thus making it possible to select node with low residual energy as $\mathrm{CH}$. This implies that such $\mathrm{CH}$ will die soon as it does not have enough energy to perform its role. In addition, presence of heterogeneity in LEACH does not significantly improve the stability of WSN, it only extend the lifespan of the network. In addition, LEACH-C uses AvE to select suitable cluster heads, but it relies on the base station to do the calculation. All sensor nodes have to forward their residual energy to the base station where AvE is calculated. Forwarding of this information to the base station adds significant energy burden to the network as sensor node expend energy sending this information. SEP protocol takes advantage of heterogeneity to improve the stability of WSN; however, selection of the cluster head is also stochastic. In SEP there is no improvement on the lifespan of the network when compared to LEACH.

The proposed algorithm is targeted at the setup phase of hierarchical routing process in order to reduce energy expended during $\mathrm{CH}$ selection. In the Setup Phase of the algorithm (i.e.CH selection), Stochastic selection technique employed in LEACH algorithm will be used to select tentative $\mathrm{CHs}$, while average energy level prediction algorithm will be used to select suitable final $\mathrm{CHs}$ from the select tentative $\mathrm{CHs}$ at any given round. Thus residual energy of sensor node is part of the criteria used to elect $\mathrm{CHs}$. The required residual energy of nodes suitable to become $\mathrm{CH}$ for current round is done by calculating the average energy ( $\mathrm{AvE}$ ) of all the nodes alive at the current round. Only nodes having residual energy equal to the average energy or higher than AvE are selected as $\mathrm{CH}$. A mathematical model is develop to estimate this AvE at each round. Assumption the each node on the network can collect information on residual energy of all other nodes on the network is avoided, since the residual energy of nodes on the network is not visible to each other. The information that is needed from the result is Average Energy of $\mathrm{CH}$ generated per round (AvE), and the Round Number (RN).

In order to extract the graph of AvE against $\mathrm{RN}$, the existing algorithm MATLAB code was modified with code below.

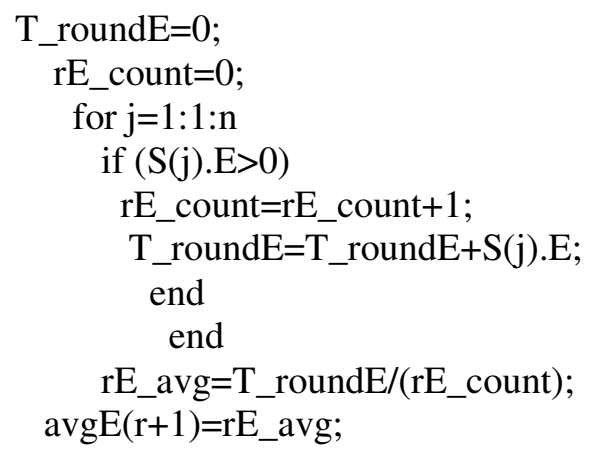

The behaviour of Average Energy of Sensor Nodes (AvE) against Round Number (RN) can be obtained from the graphical output. 
International Journal of Wireless \& Mobile Networks (IJWMN) Vol. 10, No. 5, October 2018

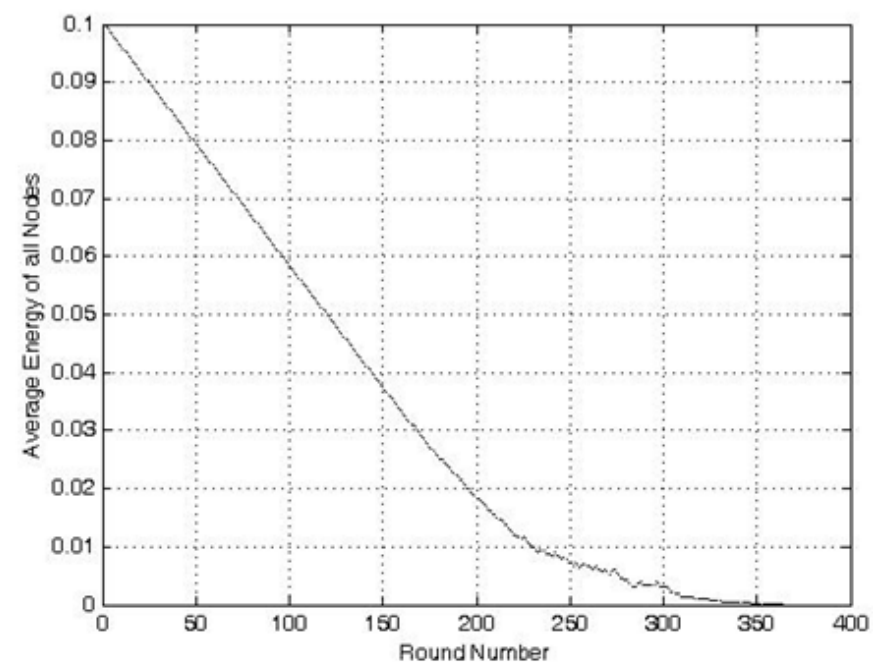

Figure 3.1 Exponential decay curve obtained for AvE with RN

\begin{tabular}{|l|l|}
\hline Description & Input Parameter \\
\hline Number of nodes & 100 \\
\hline Initial energy & $0.1 \mathrm{~J}$ \\
\hline Simulation area (field size) & $100 \mathrm{~m}^{*} 100 \mathrm{~m}$ \\
\hline Energy Consumption of Transmitting & $50 \mathrm{~nJ} / \mathrm{bit}$ \\
\hline Energy Consumption of amplifier & $10 \mathrm{pJ} / \mathrm{bit} \mathrm{m} 2$ \\
\hline Energy Consumption of data fusion & $50 \mathrm{~nJ}$.bit signal \\
\hline Packet size & 4000 bytes \\
\hline Radio propagation model & Two ray ground \\
\hline Channel Type & Wireless \\
\hline Simulation time & 500 rounds \\
\hline $\begin{array}{l}\text { Transmitter amplifier Energy } \\
\text { dissipation }\end{array}$ & $\begin{array}{l}\text { (a) Efs amp } 10 \mathrm{pJ} / \mathrm{bit} / \mathrm{m} 2 \\
\text { (b) Etray amp } 0.0013 \mathrm{~J} / \mathrm{bit} / \mathrm{m} 4\end{array}$ \\
\hline
\end{tabular}

Table 3.1 Simulation parameters used for the test environment

\subsection{Obtaining Ave From Exponential Decay Curve}

From Figure 3.1, it can be observed that the plot appears parabolic, which implies it follows exponential decay function.

The general equation for an exponential decay function is given by: $\mathrm{y}=\mathrm{Ae}^{-\mathrm{ax}}+\mathrm{B}$

From the graph in Figure 3.1

$y=\operatorname{AvE}($ Average Energy), $x=R N$ (Round Number),

After substituting "AvE" for " $y$ " and $\mathrm{RN}$ for " $\mathrm{x}$ "

We have: $\mathrm{AvE}=\mathrm{Ae}^{\mathrm{a}(\mathrm{RN})}+\mathrm{B}$ 
Where $A$ and $B$ are constant

And, $a=(1 / \mu)$ and $\mu$ is the gradient

Also, $\mu=\left(\mathrm{RN}_{2}-\mathrm{RN}_{1}\right) /\left(\right.$ in $\mathrm{AvE}_{1}-$ in $\left.\mathrm{AvE}_{2}\right)$

By taking two points in Figure 3.

$\mathrm{RN}_{2}=200, \mathrm{RN}_{1}=0, \mathrm{AvE}_{1}=0.013, \mathrm{AvE}_{2}=0.1$

After substituting the values of $R N 2, R_{1}$, in $A v E_{1}$ and in $A_{v E}$ We have:

$\mu=(200-0) /($ in $0.013-$ in 0.1$)$

$$
=-98.0248
$$

Therefore, $\mathrm{a}=(1 /-98.0248)=-0.01$

It can be implied that:

$$
\begin{aligned}
& \mathrm{AvE}_{1}=\mathrm{Ae}^{\mathrm{m}(\mathrm{RN} 1)}+\mathrm{B} \\
& \mathrm{AvE}_{2}=\mathrm{Ae}^{\mathrm{m}(\mathrm{RN} 2)}+\mathrm{B}
\end{aligned}
$$

Substituting the value of $\mathrm{AvE}, R N$ at point 1 and 2 on the graph

$$
\begin{aligned}
& 0.013=\mathrm{Ae}^{0.01(200)}+\mathrm{B} \\
& 0.1=\mathrm{Ae}^{0.01(0)}+\mathrm{B}
\end{aligned}
$$

Solving the two equations simultaneously we have

Therefore

$$
\mathrm{A}=-0.0136 \quad \mathrm{~B}=0.1136
$$

$\mathrm{AvE}=-0.0136 \mathrm{e}^{0.01 \mathrm{RN}}+0.1136$

This equation is used to modified existing LEACH-homo protocol to determine AvE suitable for $\mathrm{CHs}$ at a particular round

\section{Simulation RESUlT}

The developed algorithm was implemented on MATLAB 2013a Simulation Environment using the stated parameters in Table 3.1. Stability Period, Lifespan, and Average Energy Dissipation were used as performance metrics in the simulation result.

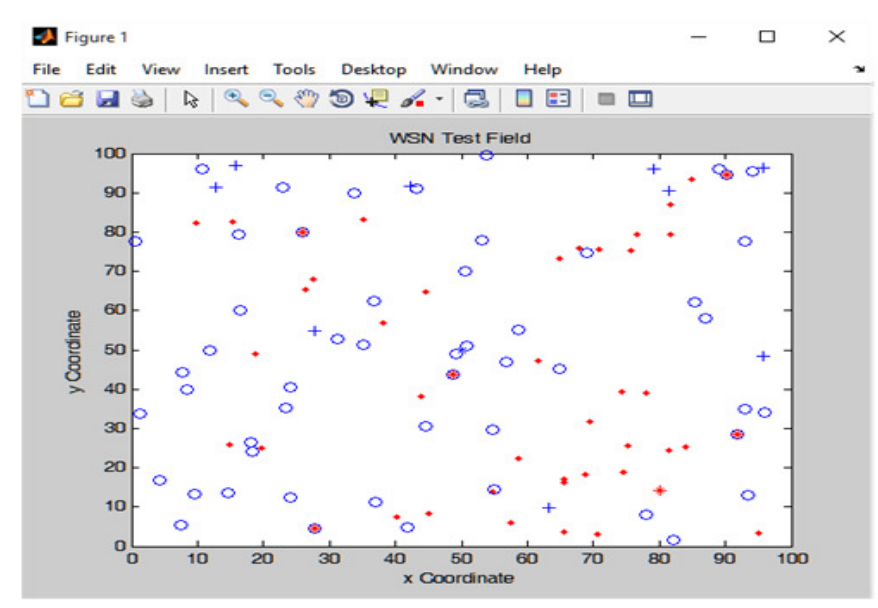

Figure 4.3. Screenshot captured showing dead and alive nodes 
International Journal of Wireless \& Mobile Networks (IJWMN) Vol. 10, No. 5, October 2018

Table 4.1. Performance analysis of energy efficiency of the developed algorithm when compared to LEACH-Homo and the Extended CHSA.

\begin{tabular}{|c|c|c|c|c|c|c|c|c|}
\hline & \multicolumn{2}{|c|}{ Alive Nodes } & \multicolumn{2}{c|}{ Dead Nodes } & \multicolumn{2}{c|}{ Average Energy (j) } & \multicolumn{2}{c|}{ Total Energy Remaining(j) } \\
\hline Round & $\begin{array}{c}\text { LEACH } \\
\text { Homo }\end{array}$ & $\begin{array}{c}\text { Extended } \\
\text { CHSA }\end{array}$ & $\begin{array}{c}\text { LEACH } \\
\text { Homo }\end{array}$ & $\begin{array}{c}\text { Extended } \\
\text { CHSA }\end{array}$ & $\begin{array}{c}\text { LEACH } \\
\text { Homo }\end{array}$ & $\begin{array}{c}\text { Extended } \\
\text { CHSA }\end{array}$ & $\begin{array}{c}\text { LEACH } \\
\text { Homo }\end{array}$ & $\begin{array}{c}\text { Extended } \\
\text { CHSA }\end{array}$ \\
\hline 50 & 100 & 100 & 0 & 0 & 0.04109 & 0.02839 & 7.9453 & 8.5806 \\
\hline 100 & 100 & 100 & 0 & 0 & 0.04197 & 0.02952 & 5.8467 & 7.1046 \\
\hline 150 & 100 & 100 & 0 & 0 & 0.04190 & 0.04124 & 3.7518 & 5.0426 \\
\hline 200 & 93 & 100 & 3 & 0 & 0.04059 & 0.04259 & 1.7223 & 2.9130 \\
\hline 250 & 40 & 83 & 60 & 17 & 0.02869 & 0.03979 & 0.2878 & 0.9235 \\
\hline 300 & 1 & 13 & 99 & 87 & 0.00572 & 0.01676 & 0.0019 & 0.0857 \\
\hline 350 & 1 & 2 & 99 & 98 & 0.00003 & 0.00160 & 0.000284 & 0.0058 \\
\hline 400 & 0 & 1 & 100 & 99 & 0.00001 & 0.00006 & 0 & 0.0029 \\
\hline 450 & 0 & 1 & 100 & 99 & 0.00000 & 0.00006 & 0 & 0 \\
\hline 500 & 0 & 0 & 100 & 100 & 0.00000 & 0.00000 & 0 & 0 \\
\hline
\end{tabular}

The results that were obtained when running the extended algorithm on homogeneous network (when $\alpha=1+0$ ), i.e. when all sensor nodes on the network has the same start up energy $E_{0}$, are as shown in following graphs and tables. At the initialization, the start-up nodes were 100 for both LEACH-Homo and ExtendedCHSA. During the simulation of the developed algorithm, the screenshot captured showing dead and alive nodes are as depicted in Figure 4.3 Alive nodes are nodes that still have residual energy and are represented using blue circle, and dead nodes are nodes that have exhausted their residual energy and are represented using red dot.

\subsection{No OF Alive NODES PER ROUND}

This shown in figure 4.1, it represent the number of alive on the network at a particular round. From the graph it can be observed that the extended algorithm is able to improve the number of Node Alive per round, due technique used in the extended cluster head selection algorithm. This also indirectly improves load balancing on the network.

Table 4.2 Performance analysis of Stability and Lifespan of LEACH-Homo and Extended LEACH

\begin{tabular}{|c|c|c|}
\hline Dead Occurrence & Leach-Homo & ExtendedCHSA \\
\hline FND & 158 & 221 \\
\hline HND & 243 & 270 \\
\hline LND & 355 & 499 \\
\hline
\end{tabular}

\subsection{Stability Period}

This shown in Table 4.2; this is the time elapse between the start of operation of the network and the time the first node dies. From the table the extended algorithm has stability period (same as FND) of 221 rounds, and that LEACH-homo is 158 rounds. This implies that the solution significantly improves the stability period of the network. 


\subsection{NO OF DEAD NODES PER ROUND}

FND, HND and LND are First Node Dead, Half Node Dead, and Last Node Dead respectively. There is significant reduction in the no of dead nodes per round the reason for this is similar as in the previous due the technique used in the cluster head selection algorithm

\subsection{DATA SEND TO CHS PER ROUND}

In figure 4.4, there is also a slight improvement in the data sent to $\mathrm{CHs}$ by MNs compared to LEACH-Homo, which is due to longer life time of the network.

\subsection{DATA SEND TO CHS PER ROUND}

In figure 4.6, there is reduction in packets of data sent to BS. However this will not have significant effect on the performance of the network, since WSN we are more concerned about the longevity of the network rather than the QoS. In addition since not all the data sent to BS are useful and the fact that the data are fused by the $\mathrm{CHs}$ to get the aggregated data, the volume of data sent to the BS should be adequate for real life operation.

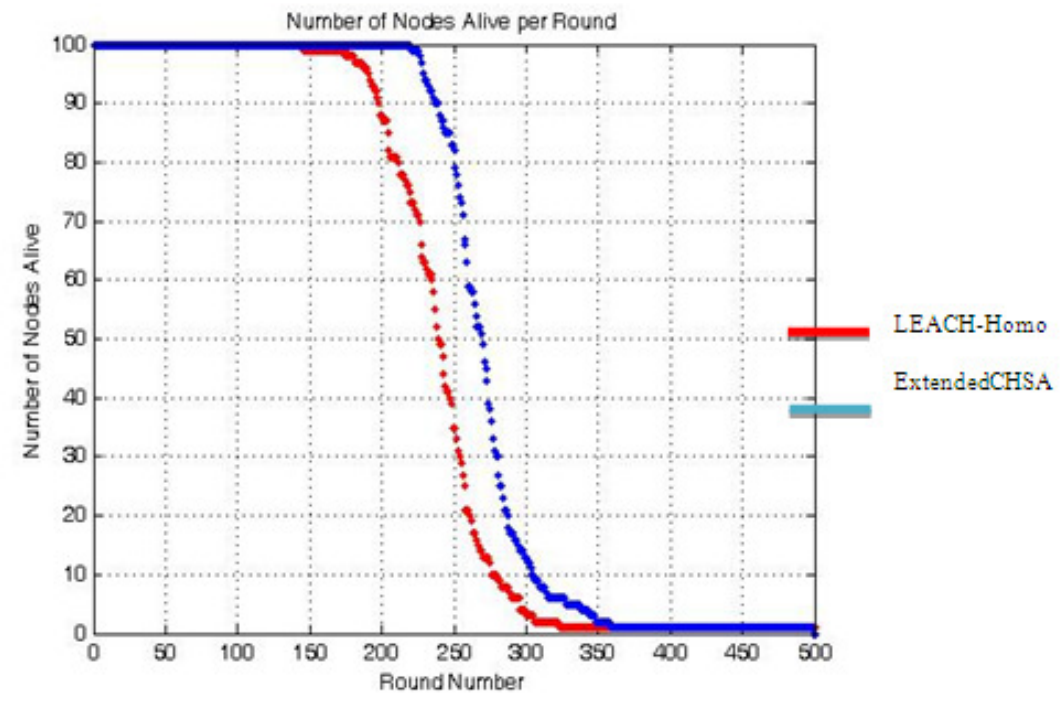

Figure 4.1 No of Alive Nodes per Round 
International Journal of Wireless \& Mobile Networks (IJWMN) Vol. 10, No. 5, October 2018

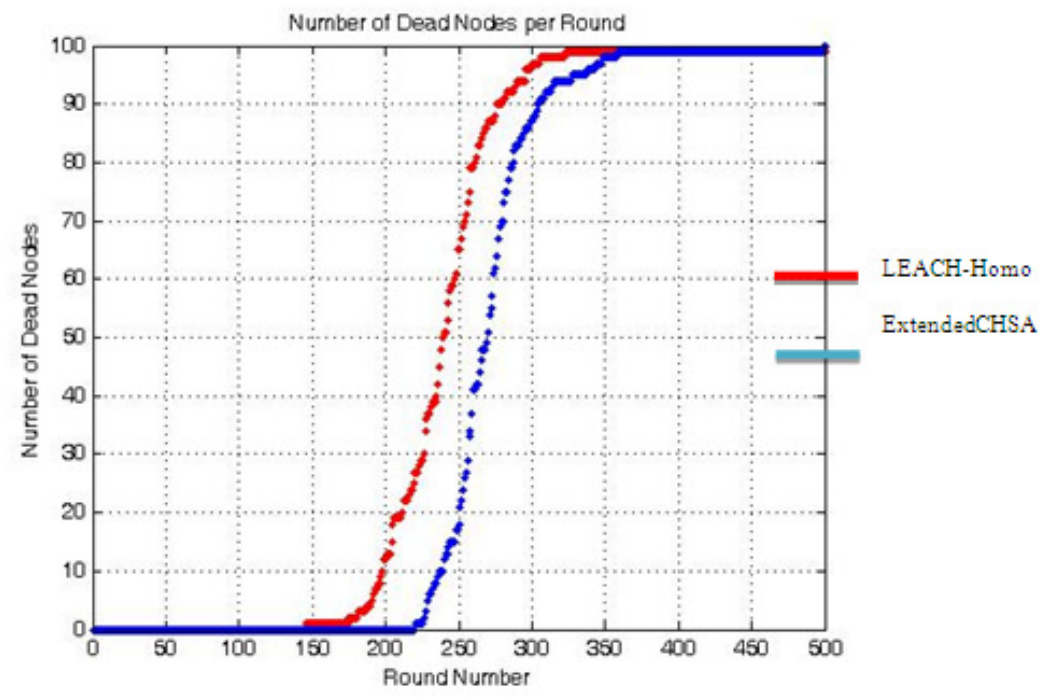

Figure 4.2 No of Dead Nodes per Round

\subsubsection{Average Energy Remaining On The Network Per Round}

As can be observed in table 4.2, there is moderate improvement in the average energy dissipation of nodes per round, with respect to the number of node currently alive on the network, when compared with LEACH-hetero. This is due to reduction in the overall energy consumption of the network per round

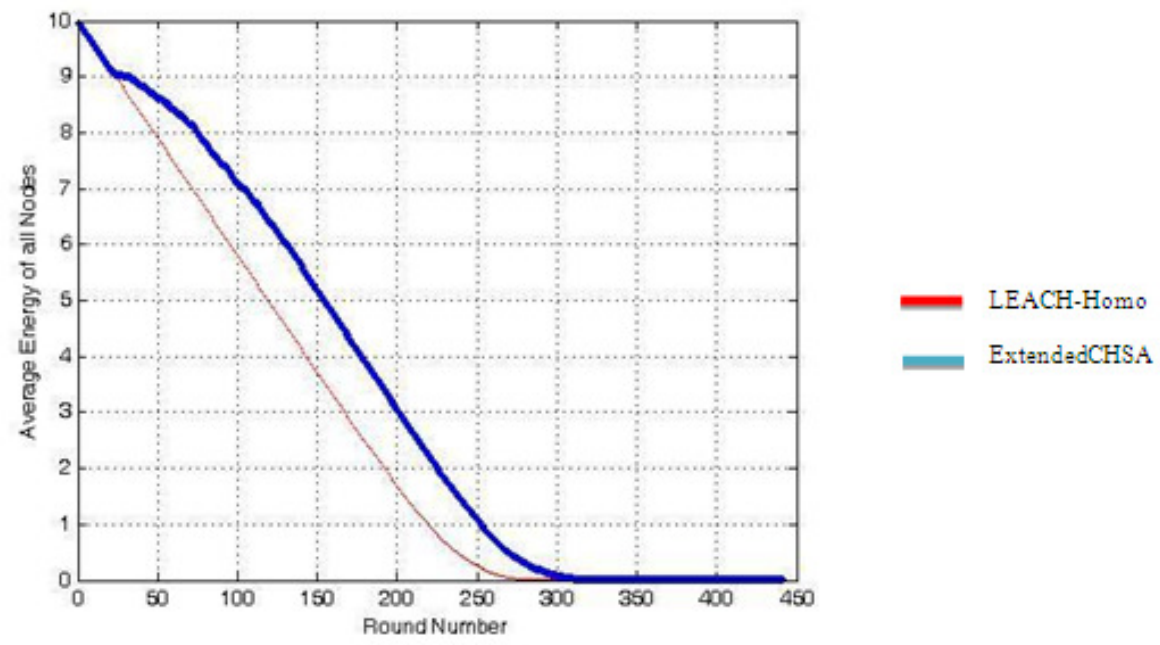

Figure 4.4 Average energy remaining per Round 


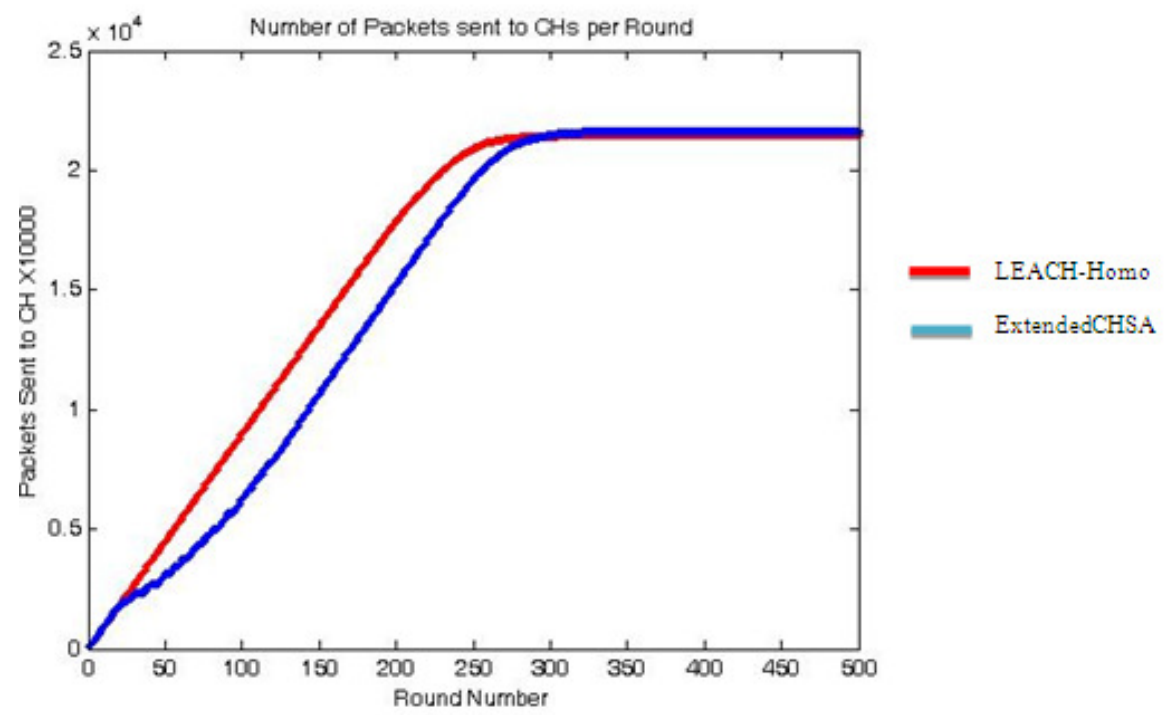

Figure 4.5 Volume of Data Sent to $\mathrm{CH}$

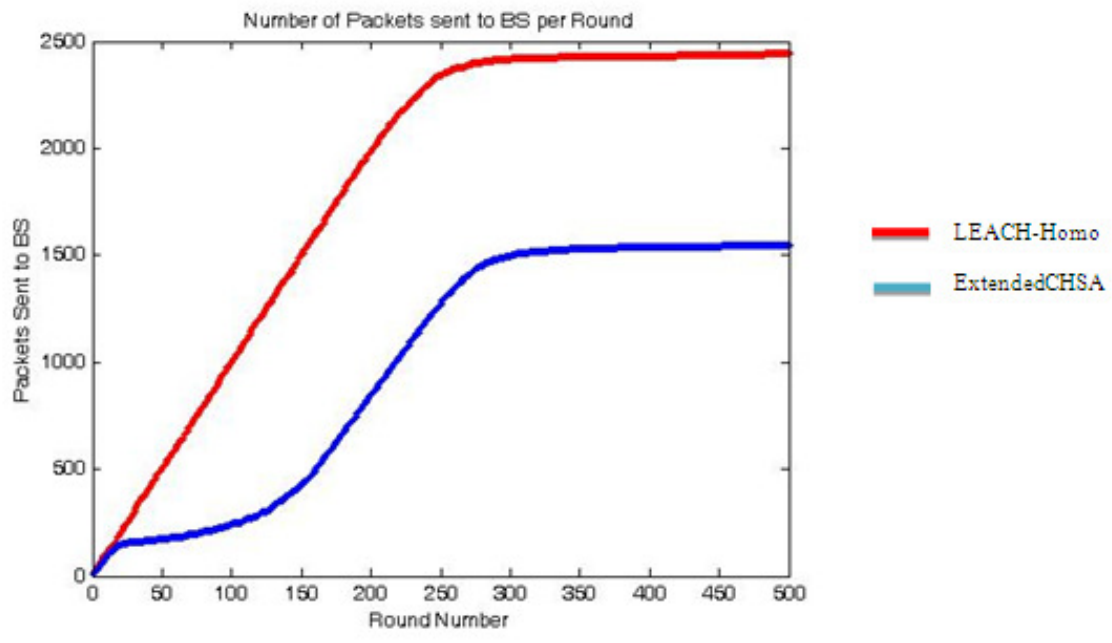

Figure 4.6 Volume of Data Sent to BS

\section{CONCLUSION}

In this research work, a technique that will be able to predict average energy of alive nodes on the network at a particular round is introduced to extend the existing method of cluster head election. Stochastic algorithm present in the existing LEACH protocol retained to maintain load balancing on the network. Making of unnecessary assumptions are also avoided, as in some existing work, cluster head election decision is taken locally without relying on the base station. In addition our extended algorithm is able to reduce energy dissipated on the network during its operation, by 
avoiding unnecessary communication. The developed algorithm was simulated on MATLAB 2013 simulation environment using parameters earlier stated, we then compare the result obtained with the selected hierarchical routing protocol using the following performance metrics, Stability period, lifespan, and Average energy dissipation per round, Simulation result from the study shows that for homogeneous, the developed algorithm is able to extend the stability of the network, lifespan of the network and average energy dissipated at each round of the operation of the network when compared with LEACH-Homo.

\section{REFERENCES}

[1] Loscrì G. Morabito and S. Marano. (2005). A Two- Levels Hierarchy for Low-Energy Adaptive Clustering Hierarchy, Vehicular Technology Conference, VTC-2005-Fall. IEEE 62nd. 1809-1813

[2] Yassein M. B., Al-zou'bi A., Khamayseh Y and Mardini W. (2009). Improvement on LEACH Protocol of Wireless Sensor Network (VLEACH), International Journal of Digital Content Technology and its Applications, 3 (2), 132-136.

[3] Heinzelman W.R., Chandrakasan A.P., Balakrishnan, H., (2002). An application-Specific Protocol Architecture for Wireless Microsensor Networks, IEEE Transactions on Wireless Communications, 1 (4) 660-670.

[4] Tong M. and Tang, M. (2010). LEACH-B: An Improved LEACH Protocol for Wireless Sensor Network, Wireless Communications Networking and Mobile Computing (WiCOM), 6th International Conference.

[5] Nguyen D. T., Longzhe H., Nguyen D. V., and Minho J., (2012). An Improved LEACH Routing Protocol for Energy-Efficiency of Wireless Sensor Networks, Smart Computing Review, 2 (5)

[6] Heinzelman W.R, Chandrakasan A, and Balakrishnan H., (2000). Energy Efficient Communication Protocol for wireless Microsensor Networks. Proceedings of the 33rd Hawaii International Conference on System Sciences.

[7] Smaragdakis G. and Matta I. (2004), SEP: A Stable Election Protocol for Clustered Heterogeneous Wireless Sensor Networks. Retrieved from: http://www.cs.bu.edu/techreports/pdf/2004-022-sep.pdf

[8] Xiangning Fan, Yulin Song, (2007). Improvement on LEACH protocol of Wireless Sensor Networks. International Conference on Sensor Technologies and Applications.

[9] Wang W., Wang Q., Wei Luo, Sheng M. Wu W., Hao L., (2009). Leach-H: An Improved Routing Protocol for Collaborative Sensing Networks, International conference on Wireless Communications \& Signal Processing.

[10] Majadi N., "U-LEACH: (2012). A Routing Protocol for Prolonging Lifetime of Wireless Sensor Networks, International Journal of Engineering Research and Applications (IJERA) ISSN: 2248-9622 2 (4), 1649-1652. Retrieved from: https://research.ijcaonline.org /ccsn/number4/ccsn028.pdf

[11] Prakashgoud P., Umakant K, Ayachit N. H., (2011). Some Issues in Clustering Algorithms for Wireless Sensor Networks IJCA Special Issue on "2nd National Conference- Computing, Communication and Sensor Network" CCSN, 18. Retrieved from: https://research.ijcaonline.org/ccsn/number4/ccsn028.pdf 
International Journal of Wireless \& Mobile Networks (IJWMN) Vol. 10, No. 5, October 2018

[12] Qing L., Zhu Q., Wang M., (2010). Design of a distributed energy-efficient clustering algorithm for heterogeneous wireless sensor networks". ELSEVIER, Computer Communications 29, pp 2230-2237.

[13] Xuxun L, (2012). A Survey on Clustering Routing Protocols in Wireless Sensor Networks. Retrieved from www.mdpi.com/journal/sensors. ISSN 1424-8220

[14] Kumar P., Singh M.P., and Triar U.S., (2012), A Review of Routing Protocols in Wireless Sensor Network. International Journal of Engineering Research \& Technology(IJERT), 1 (4), pp. 1-14

\section{Authors}

Ojoawo, Akinwale Olusola received his B.Tech Degree from Ladoke Akintola University of Technologies, LAUTECH, Ogbomoso, in 2001. He has just concluded his M.Sc Computer Science at University of Ibadan, Ibadan. Nigeria. He is currently lecturing at Computer science department, The Ibarapa Polytechnic, Eruwa, Nigeria. He has a number of publications in various national/international journals and conferences. His current research areas are wireless sensor network and mobile ad hoc network.

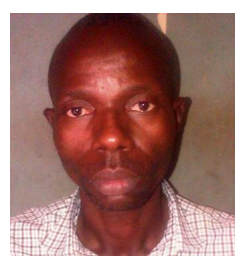

